Revue

Revue de l'histoire des religions

del'histoire des religions

\title{
Entre Bousiris et Abydos, la (dé)rive de Nédit : mythologie égyptienne et dualisme géographique
}

\section{Laurent Coulon}

\section{QpenEdition \\ Journals}

Édition électronique

URL : http://journals.openedition.org/rhr/9348

DOI : $10.4000 /$ rhr.9348

ISSN : 2105-2573

Éditeur

Armand Colin

Édition imprimée

Date de publication : 1 décembre 2018

Pagination : 683-699

ISBN : 978-2-200-93200-8

ISSN : 0035-1423

Référence électronique

Laurent Coulon, «Entre Bousiris et Abydos, la (dé)rive de Nédit : mythologie égyptienne et dualisme géographique », Revue de l'histoire des religions [En ligne], 4 | 2018, mis en ligne le 01 décembre 2020, consulté le 13 janvier 2021. URL : http://journals.openedition.org/rhr/9348 ; DOI : https://doi.org/ 10.4000/rhr.9348 


\title{
Entre Bousiris et Abydos, la (dé)rive de Nédit : mythologie égyptienne et dualisme géographique
}

\begin{abstract}
Mythologie et géographie sont indissociablement liées en Égypte ancienne, par le seul fait que les mythes sont souvent des récits étiologiques sur ses composantes territoriales. Cette contribution aborde le cas particulier de Nédit, qui désigne dans les sources égyptiennes le lieu où Osiris a été tué par Seth et dont la localisation a été l'objet de vifs débats. Des données concordantes permettraient de le situer dans le Delta oriental, non loin de Bousiris, d'autres autorisent à établir une équivalence avec Abydos. L'acclimatation à des fins rituelles du toponyme ne suffit pas à rendre compte d'une délocalisation qui impliquait une adaptation du mythe lui-même. L'analyse des récits mythologiques des papyrus Jumilhac et Salt 825 permet de mieux comprendre l'usage qui est fait du toponyme.
\end{abstract}

The wandering of Nedit, between Busiris and Abydos: Egyptian mythology and geographical dualism

Mythology and geography are inextricably linked in ancient Egypt, by the mere fact that its myths are often aetiological narratives about its territorial components. This contribution focuses on Nedit, a toponym which, in ancient Egyptian sources, designates the place where Osiris was killed by Seth. Its location has been the subject of much debate. Consistent evidence would make it possible to locate it in the Eastern Delta, not far from Busiris, but an equivalence with Abydos is another possibility. The transfer of the toponym for ritual purposes is not a suitable explanation to account for a delocalization that would imply an adaptation of the myth itself. The analysis of the mythological accounts from papyri Jumilhac and Salt 825 allows a better understanding of the use of the toponym. 
Les mythes égyptiens nous sont fréquemment accessibles à travers un discours étiologique, qui rattache à un toponyme un récit qui l'enracine dans un précédent mythologique, par le biais d'un jeu de mots ${ }^{1}$. Réciproquement, le toponyme peut «encapsuler» une série de mythèmes, qui sont en quelque sorte implicitement mobilisés à sa simple évocation. Il existe même des toponymes qui ne nous sont connus que par leur «emploi mythologique», au point que leur localisation reste imprécise ${ }^{2}$ ou peut être démultipliée dans différentes parties d'Égypte. L'étude qui suit est centrée sur l'un d'entre eux, Nédit, lieu du meurtre d'Osiris, dont la localisation semble osciller entre Bousiris et Abydos selon les adaptations théologiques des hiérogrammates et en fonction d'un dualisme qui est fondamental dans le système de pensée égyptien ${ }^{3}$, autant qu'elle parait dériver au gré des interprétations égyptologiques désireuses de fixer dans l'espace «réel» des épisodes mythologiques qui ne leur offrent pourtant que peu d'indices topographiques.

\section{NÉdit ET LE CONTEXTE BOUSIRITE}

Nédit est principalement connu pour désigner le lieu funeste où Osiris aurait été assassiné par son frère Seth, avant d'y être retrouvé

1. Cf. e.g. Joachim Fr. Quack, «Lokalressourcen oder Zentraltheologie? Zur Relevanz und Situierung geographisch strukturierter Mythologie im Alten Ägypten », Archiv für Religionsgeschichte, t. 10, 2008, p. 12-13.

2. Voir dernièrement à propos de Naref la monographie de Lucía DíazIglesias Llanos, Naref and Osiris Naref. A Study of Herakleopolitan Religious Traditions, Berlin-Boston, de Gruyter («Zeitschrift für ägyptische Sprache und Altertumskunde Beihefte», t. 3), 2017, part. p. 27-35.

3. Eberhard Otto, Lexikon der Ägyptologie, éd. Wolfgang Heck, Eberhard Otto, Wiesbaden, Harrassowitz, t. I, 1975, s.v. « Dualismus », col. 1148-1150 ; Frédéric Servajean, «Duality », éd. Jacco Dieleman, Willeke Wendrich, UCLA Encyclopedia of Egyptology, Los Angeles, consulté le 2 août 2018. URL : http:// digital2.library.ucla.edu/viewItem.do?ark=21198/zz0013x9jp ; Ivan Guermeur, «Du dualisme et de l'ambivalence séthienne dans la pensée religieuse de l'Égypte ancienne », Dualismes, "Doctrines religieuses et traditions philosophiques », éd. Fabienne Jourdan, Anca Vasiliu, Hors-Série de la revue $\chi \omega ́ \rho \alpha$, Bucarest, Polirom edition, 2015, p. 63-88. 
par ses sœurs Isis et Nephthys ${ }^{4}$. Il peut aussi, plus rarement, servir de cadre à d'autres épisodes mythologiques tragiques 5 . Le tabou qui entoure ce qui concerne la mort d'Osiris entraîne une discrétion des sources concernant la scène de crime ${ }^{6}$. Les Textes des Pyramides évoquent à tout le moins le meurtre d'Osiris et la découverte de son cadavre à Nédit ou «sur la rive de Nédit » ${ }^{7}$, mentionnant parallèlement le toponyme Géhesty pour situer ce décès ${ }^{8}$. Le toponyme Nédit fait l'objet d'un jeu de mots étymologique à partir de l'expression $n d j r$ t3 «jeter à terre $»^{9}$, mais n'a pas de réalité

4. Cf. Pascal Vernus, «Le Mythe d'un mythe : la prétendue noyade d'Osiris. De la dérive d'un corps à la dérive du sens », Studi di egittologia e di Antichità puniche, t. 9, 1991, p. 19-20; Jan Assmann, avec la collaboration de Martin Bommas, Altägyptische Totenliturgien, t. I. Totenliturgien in den Sargtexten des Mittleren Reiches, Heidelberg, C. Winter, 2002, p. 383-386.

5. Voir l'allusion au combat d'Horus et de Seth à Nédit en Coffin Texts V, $128 \mathrm{a}$, et, de manière plus précise, la localisation à cet endroit de l'épisode des mains coupées d'Horus dans le papyrus médical Louvre E 32847, Rx+16, 1-3 : « Rê en personne est en deuil à cause des dieux d'Héliopolis qui sont en conflit. Hathor est en train de pleurer car Horus a jeté à terre ses mains à cause du harponneur de la barque sacrée sur ce banc de sable de Nédit » (trad. Thierry Bardinet, Médecins et magiciens à la cour du pharaon, Paris, éditions du Louvre-Khéops, 2018, p. 320-321). Sur cet épisode, voir Youri Volokhine, « Reliques et traces en Égypte ancienne. À propos de la présence sur terre d'écrits et d'objets d'origine divine », Les objets de la mémoire. Pour une approche comparatiste des reliques et de leur culte, éd. Philippe Borgeaud, Youri Volokhine, Berne, Peter Lang ( Studia Religiosa Helvetica », t. 2004/05), 2005, p. 68-70.

6. Voir Laurent Coulon, "Osiris chez Hérodote», Hérodote et l'Égypte. Regards croisés sur le livre II de l'Enquête d'Hérodote, éd. Laurent Coulon, Pascale Giovannelli-Jouanna, Flore Kimmel-Clauzet, Lyon, Maison de l'Orient et de la Méditerranée, ( Collection de la Maison de l'Orient et de la Méditerranée », t. 51), 2013, p. 173-177 (avec réf.).

7. Cf. Thesaurus Linguae Aegyptiae, lemme $\mathrm{n}^{\circ} 90710$; voir Georg Meurer, Die Feinde des Königs in den Pyramidentexten, Fribourg-Göttingen, Universitätsverlag et Vandenhoeck \& Ruprecht (« Orbis Biblicus et Orientalis », t. 189), 2002, p. 122-125.

8. Pour Géhesty, lit. «la place des deux gazelles », voir G. Meurer, Die Feinde des Königs in den Pyramidentexten, p. 124-125; Åsa Strandberg, The Gazelle in Ancient Egyptian Art. Image and meaning, Uppsala, Uppsala University (« Uppsala Studies in Egyptology », t. 6), 2009, p. 162-170.

9. Pyramid Texts, Spell 532, § 1256a-b : gm.n.sn Wsjr ndj.n sw sn.f sťs $r$ t3 $m$ $N d j t:$ «Elles (= Isis et Nephthys) trouvèrent Osiris que son frère Seth avait jeté à terre dans Nédyt ». Pour ce jeu de mots, voir aussi le commentaire de P. Vernus, «Le Mythe d'un mythe : la prétendue noyade d'Osiris », p. 19, (1)-(2a). Voir aussi Papyrus Jumilhac XI, 20-21 et ibid., p. 20 (10) et 28, n. 15. Un jeu de mots est également employé pour lier le toponyme Géhesty à la mort d'Osiris, dont on précise qu'il a été trouvé gisant « sur son flanc » (hr gs.f). Voir e.g. Pyramid Texts, Spell 485, § 1033b. Sur le vocabulaire décrivant les circonstances du meurtre d'Osiris dans les Textes des Pyramides, voir Bernard Mathieu, «Mais qui est 
géographique très définie ${ }^{10}$. Néanmoins, certains éléments de contexte peuvent être déduits de quelques sources textuelles qui inciteraient à situer Nédit non loin de Bousiris, quelque part dans le Delta oriental ${ }^{11}$. Ainsi, dans les «veillées horaires d'Osiris» (Stundenwachen), Isis déclare qu'elle a découvert le corps d'Osiris « sur cette rive nord de Bousiris » $(h r w \underline{d} b \text { pfy mhty } \underline{D} d w)^{12}$, ce qui apparaît comme une paraphrase de «sur la rive de Nédit $»^{13}$. Par ailleurs, le toponyme est mentionné dans les textes géographiques relatifs aux $8^{\mathrm{e}}$ (Harpon oriental) et $14^{\mathrm{e}}$ (tanitique) nomes de Basse Égypte, ce qui a pu laisser penser qu'il renvoyait à une frontière commune aux deux districts ${ }^{14}$. Quant à la version du mythe osirien

donc Osiris ? Ou la politique sous le linceul de la religion », Égypte Nilotique et Méditerranéenne, t. 3, 2010, p. 77-107, ici p. 97.

10. Wolfgang Helck, « Topographisierung von Begriffen und Gegenständen in den Pyramidentexten », Hommages à François Daumas, Montpellier, Publication de la recherche, 1986, t. 2, p. 422-423 ; Christoffer Theis, Magie und Raum. Der magische Schutz ausgewählter Räume im alten Ägypten nebst einem Vergleich $z u$ angrenzenden Kulturbereichen, Tübingen, Mohr Siebeck ( Orientalische Religionen in der Antike Ägypten, Israel, Alter Orient », t. 13), 2014, p. 446.

11. Voir les références dans François-René Herbin, Le livre de parcourir l'éternité, Louvain, Peeters («Orientalia lovaniensa analecta», t. 58), 1994, p. 182 ; G. Meurer, Die Feinde des Königs in den Pyramidentexten, p. 122 ; Dimitri Meeks, Mythes et légendes du Delta d'après le papyrus Brooklyn 47.218.84, Le Caire, Institut français d'archéologie orientale, ( Mémoires publiés par les membres de l'Institut français d'archéologie orientale », t. 125), 2006, p. 182.

12. Andreas Pries, Die Stundenwachen im Osiriskult. Eine Studie zur Tradition und späten Rezeption von Ritualen im Alten Ägypten, Wiesbaden, Harrassowitz ( «Studien zur Spätägyptischen Religion», t. 2), 2011, t. I, p. 161 et t. II, p. 33 (02 NS $\sigma E 14)$. Comme le rappelle A. Pries (ibid., t. I, p. 161, n. 648), la première édition de Hermann Junker pâtissait d'une surprenante confusion entre Bousiris et Abydos qui amenait à la traduction : " auf jenem nördlichen Ufer von Abydos » (Hermann Junker, Die Stundenwachen in den Osirismysterien nach den Inschriften von Dendera, Edfu und Philae, Vienne, Alfred Hölder, 1910, p. 84) ; mais la traduction correcte a été fournie par le même auteur dans Hermann Junker, Die Politische Lehre von Memphis, Berlin, Verlag der Akademie der Wissenchaften, 1941, p. 52.

13. Pour le contexte bousirite de Nédit, voir aussi un autre passage des Stundenwachen qui mentionne conjointement les «berges de Nédit» (hryw $n$ Ndjt), Bousiris et son nome: A. Pries, Die Stundenwachen im Osiriskult, t. I, p. 366 ; t. II, p. 96 (02 TS $\lambda$ E9).

14. Cf. Henri Gauthier, Dictionnaire des noms géographiques contenus dans les textes hiéroglyphiques, Le Caire, Société royale de géographie d'Égypte, 1925-1931, t. III, p. 111 ; Christian Leitz, Geographisch-osirianische Prozessionen aus Philae, Dendara und Athribis. Soubassementstudien, Wiesbaden, Harrassowitz («Studien zur Spätägyptischen Religion », t. 8), 2012, t. II, p. 405-408. Les textes géographiques relatifs au nome tanitique (Hnty-j3btt) évoquent le «phénix (bnw/bng) dans Nedjyt (= Nédit)». Voir aussi Christiane 
fournie par Plutarque dans son De Iside et Osiride, elle indique qu'Osiris, dupé par Seth et ses alliés, fut enfermé dans un coffre puis jeté au Nil afin qu'il rejoignît ensuite la mer par la branche tanitique, ce qui est cohérent avec les indications précédentes ${ }^{15}$.

Le témoignage des inscriptions de la Cuve de Coptos, d'époque libyenne, vient étayer l'ancrage de Nédit dans un contexte résolument bousirite, puisque le toponyme est mentionné au sein de rituels dont l'horizon géographique ne dépasse que rarement cette région ${ }^{16}$. Des « appels rituels s'adressant à Osiris » (col. VI, 34-40) font intervenir «deux formes de Khnoum associées au lieu où échoua le corps de ce dieu »: Khnoum qui réside à Nédit

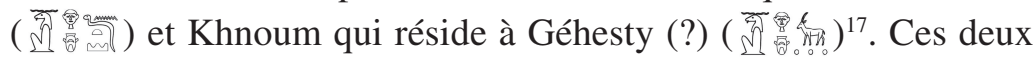

Zivie-Coche, «Khentetiabtet. L'invention d'une déesse tout orientale », Et in Aegypto et ad Aegyptum. Recueil d'études dédiées à Jean-Claude Grenier, éd. Annie Gasse, Frédéric Servajean, Christophe Thiers, Montpellier, Université Paul Valéry («Cahiers de l'Égypte Nilotique et Méditerranéenne», t. 5), 2012, p. 784-785 et n. 28.

15. Plutarque, De Iside et Osiride, § 13 (356B-C). Sur cet épisode, voir récemment Pierre Koemoth, « Du Nil à Byblos : de la dérive du corps au périple maritime du roi Osiris», Bibliotheca Orientalis, t. 67, 2010, col. 461-488. Plutarque précise que la branche tanitique en avait acquis une réputation de branche «maudite », ce qui s'accorde assez bien avec la sombre image de Nédit. Cf. e.g. Coffin Texts VII, $26 \mathrm{t}$ : «Il n'est pas question que je me purifie dans l'eau de Nédit. » (cité par P. Vernus, «Le Mythe d'un mythe : la prétendue noyade d'Osiris », p. 29, n. 19).

16. Jean Yoyotte, «Religion de l'Égypte ancienne. I. La cuve osirienne de Coptos », Annuaire de l'EPHE Sciences Religieuses, t. 86, 1977-1978, p. $168=$ Opera Selecta, Louvain, Peeters («Orientalia lovaniensa analecta », t. 224), 2013 p. 390 : «En récapitulant l'ensemble des données géographiques fournies par les inscriptions de la cuve, on constate que les rites en cours se déroulent dans divers lieux saints de la seule Busiris : la «maison » ou «temple» d'Osiris, dénommé aussi «le Temple Saint» [...], le sanctuaire et le téménos de Shentayt, et un monument appelé « le Château de la Mesnakhté »; le passage VI, 35-39 prouverait que la localisation de Nédit sur la haute rive de Busiris était admise dès les hautes époques. Pour le reste, l'horizon géographique ne s'étend au-delà du Delta oriental (Mendès, Athribis, Pharbaethos) que lorsqu'on évoque la présence universelle de la Shentayt locale - dite aussi « la Maîtresse de la Ville » et la Mesnakhté, «la revigoreuse »- qui se confond avec Hathor, avec l'Or. »

17. J. Yoyotte, «Religion de l'Égypte ancienne. I. La cuve osirienne de Coptos », p. 190 (= Opera Selecta, p. 404). Les transcriptions hiéroglyphiques ont été ajoutées par nous en s'appuyant sur les relevés épigraphiques et commentaires sur la Cuve de Coptos conservées dans les archives Jean Yoyotte [boîte ${ }^{\circ}{ }^{19}$ ] au Centre Wladimir Golénischeff, ÉPHÉ, Paris. Les propos de J. Yoyotte dans son compte rendu comme ses notes de travail trahissent le caractère incertain de sa lecture «Gehesty», le mammifère servant à écrire le toponyme n'ayant pas de caractère distinctif très marqué. 
Khnoum jouent un rôle vis-à-vis de l'Osiris assassiné, que l'état du texte, presque indéchiffrable à certains endroits, ne permet pas de préciser. Il est toutefois exceptionnel d'avoir ici une épiclèse divine renvoyant à Nédit comme s'il s'agissait a priori d'un lieu de culte ${ }^{18}$. Le dualisme établi entre Nédit et Géhesty a probablement joué un rôle dans l'utilisation de cette désignation, car le culte de Khnoum maître de Géhesty est, lui, bien attesté, notamment par des titres de prêtrises de la $\mathrm{XXI}^{\mathrm{e}}$ dynastie, et ce toponyme, à défaut d'être localisé de manière certaine, a une réalité géographique mieux circonscrite ${ }^{19}$. Ces deux Khnoum en évoquent deux autres, dans un contexte abydénien cette fois, à savoir les dieux-béliers qui encadrent traditionnellement le fétiche d'Abydos, Khnoum de la Cataracte et Khnoum créateur, maître d'Her-Our/Hout-ouret ${ }^{20}$.

Dans le Livre de Parcourir l'éternité, liturgie attestée par des sources d'époque ptolémaïque et romaine qui offre un large panorama des rites et croyances des localités d'Égypte que le défunt est censé parcourir, le toponyme Nédit est attesté dans un contexte théologique clairement bousirite :

18. L'expression jmy Ndjt «celui qui est dans Nédit» (Lexikon der ägyptischen Götter und Götterbezeichnungen, Christian Leitz éd., Louvain, Peeters [ "Orientalia lovaniensa analecta », t. 110], I, 240b) qui désigne régulièrement Osiris dans les textes funéraires n'a pas ce statut d'épiclèse, mais plutôt celui d'antonomase.

19. A. Strandberg, The Gazelle in Ancient Egyptian Art, p. 166-167 et 170.

20. Cf. Laurent Coulon, «Les uraei gardiens du fétiche abydénien. Un motif osirien et sa diffusion à l'époque saïte », La XXVI dynastie. Continuités et ruptures. Actes du colloque de l'Université de Lille-III. 26-27 novembre 2004, éd. Didier Devauchelle, Paris, Cybèle, 2011, p. 98, n. (q). Sur le rôle de ces divinités, ajouter pTurin CG 54050 rt. 3,1 (éd. Roccati), avec le commentaire de Joris F. Borghouts, The Magical Texts of Papyrus Leiden I 348, Leyde, Brill («Oudheidkundige Mededelingen vit het Rijksmuseum van Oudheden », t. LI), 1971, p. 116-117, n. 248. Notons aussi que Khnoum maître de Hout-ouret intervient dans un épisode du «Texte B » des stèles d'Horus sur les crocodiles qui a pour cadre Nédit. Il menace ainsi le crocodile : «Détourne de moi tes pas, ennemi ! Je suis Khnoum, maître de Hout-our. Garde-toi de renouveler ton crime en une seconde fois à cause de ce qui t'a été fait en présence de la Grande Ennéade. Écarte-toi donc ! Hy ! hy ! Je suis le fils de Sekhmet enfanté par Isis. Hy ! déesse, hy ! hy ! Tu n'as donc pas entendu ma voix au moment de l'obscurité sur cette rive de Nedyt (Nédit), le grand cri de chaque dieu, de chaque déesse, les grands dieux étant en lamentation (à cause) du grand dommage que t'a fait l'ennemi. Rê est en rage et en furie à cause de cela. Il ordonne que l'on fasse ton massacre. » (trad. Annie Gasse, "Une stèle d'Horus sur les crocodiles. À propos du "texte C" », Revue d'Égyptologie, t. 55, 2004, p. 27). 
«Ton $b a$ s'élance vers les rives de Nédit $(r w \underline{d} b w n w N \underline{d} y t)$, le jour de la grande fête de recouvrir la tête.

Tu entends le son des chants des deux pleureuses gémissantes, le jour de la fête du grand deuil ${ }^{21} »$.

La mention de la cérémonie du hobs tp (fête de recouvrir la tête) fait écho au rôle du prêtre $h b s$ tp, évoqué ailleurs dans le papyrus (VI, 22). Il est attesté dans le chapitre 168 du Livre des Morts dans un contexte là encore bousirite : «Dresse-toi pilier- $\underline{d} d$ à Bousiris ! Le prêtre $h b s$-tp a caché tes formes » (hbs-tp jmn.n.f $j r w . k)^{22}$.

De fait, dans d'autres passages du Livre de parcourir l'éternité, Nédit est convoqué, selon l'éditeur, pour servir de pendant géographique dans l'opposition symétrique Bousiris / Abydos :

«Tu survoles la terre sur la rive de Nédit ( $h r w \underline{d} b n N \underline{d} y t)$, et te promènes dans le Château-des-aliments $\left(h w t-\underline{d} f_{3} w\right)^{23} »$.

Le Château-des-aliments, sur lequel nous reviendrons plus bas, est un toponyme abydénien. Cette mise en symétrie des lieux saints osiriens intervient à plusieurs reprises, ce qui tendrait à confirmer la dualité supposée entre Nédit / Bousiris d'une part, Abydos et ses lieux sacrés d'autre part :

« Tu vogues vers Bousiris, tu navigues vers Ta-our,

(de sorte) que les provinces divines possèdent ton image.

Tu accèdes à la barque-nšmt en compagnie des bienheureux, et ta place est à l'avant dans la barque divine.

Tu abordes les berges de Ândjty (= Bousiris) et atteins les rivages de Hapennebes (= nécropole d'Abydos) ${ }^{24} »$.

21. Papyrus Leyde T 32, V, 13-14 ; Fr.-R. Herbin, Le livre de parcourir l'éternité, p. 61 et 202.

22. Fr.-R. Herbin, Le livre de parcourir l'éternité, p. 228-229. L'incertitude qui pèse sur la localisation de Nédit amène néanmoins des considérations prudentes de l'auteur sur la possibilité d'un lien avec la relique abydénienne de la tête (ibid., p. 314).

23. Papyrus Leyde T 32, IV, 13-14 ; Fr.-R. Herbin, Le livre de parcourir l'éternité, p. 58 et 182 ; Mark Smith, Traversing Eternity. Texts for the Afterlife from Ptolemaic and Roman Egypt, New York, Oxford University Press, 2009, p. 417.

24. Papyrus Leyde T 32, I, 15-17 ; Fr.-R. Herbin, Le livre de parcourir l'éternité, p. 48 et 98-101. 


\section{Nédit et Abydos}

L'identification de Nédit à un toponyme deltaïque pourrait sembler affaire entendue. Néanmoins, il a été également proposé - et cela est couramment défendu dans la littérature égyptologique ${ }^{25}$ - que Nédit était un toponyme authentiquement abydénien. Ainsi John Gwyn Griffiths a contesté le lien entre Nédit et Bousiris en arguant du fait qu'Abydos est le lieu du tombeau principal d'Osiris et en citant le passage suivant des Textes des Pyramides: "Le transfiguré de Nédit est venu, la puissance de Ta-our» (j 3hj pw jmj ndjt shm jmj t3-wr $)^{26}$. Pour Griffiths, il y aurait équivalence entre les deux expressions et donc équation entre Nédit et Ta-our, le nome thinite ${ }^{27}$. Nous touchons là un problème central de l'interprétation des textes égyptiens fondés sur le parallelismus membrorum: les deux termes peuvent être en symétrie synonymique ou en équivalence complémentaire. Les travaux d'Otto Firchow sur la stylistique des Textes des Pyramides ont montré la diversité des cas de figure possibles dans les paires sémantiques qui structurent en permanence les discours liturgiques: parallélisme d'identité, d'antithèse, d'équivalence. Si l'on admet que Nédit est près de Bousiris, on aurait entre Nédit et Ta-our ce que Firchow appelle un parallélisme d'équivalence par corrélation géographique ${ }^{28}$. Il est aisé d'objecter que des formulations voisines dans d'autres sources obligeraient à d'autres équivalences. Ainsi dans les Stundenwachen, Osiris se voit interpeller ainsi : «Dressetoi, grande puissance dans Nédit, dieu vénérable qui préside à Rô-Setaou! $»^{29}$. Le raisonnement de Griffiths impliquerait logiquement d'identifier Nédit au toponyme memphite dans ce cas, ce qui amène à une impasse tant les possibilités sont

25. E.g. Marc Étienne éd., Les portes du ciel. Visions du monde dans l'Égypte ancienne, Paris, Musée du Louvre-Somogy, 2013, p. 68.

26. Pyramid Texts, $422 \S 754 \mathrm{c}$.

27. Voir en dernier lieu John Gwyn Griffiths, The Origins of Osiris and his Cult, Leyde, Brill, 1980, p. 22. Pour des opinions antérieures similaires, voir G. Meurer, Die Feinde des Königs in den Pyramidentexten, p. 123, n. 4.

28. Otto Firchow, Gründzüge der Stilistik in den altägyptischen Pyramidentexten, Berlin, Akademie Verlag, 1953, p. 103-105.

29. A. Pries, Die Stundenwachen im Osiriskult, t. I, p. 388 ; t. II, p. 103 (04 TS $\eta E 17)$. 
nombreuses ${ }^{30}$. Griffiths mobilise néanmoins à l'appui de son affirmation le texte de la célèbre stèle d'Ikhernofret dans laquelle il raconte comment, sous le règne de Sésostris III, il a conduit les mystères d'Osiris à Abydos :

« J'ai sanctifié les chemins du dieu vers sa tombe dans Ou-Peker (Abydos). J'ai protégé Ounnenefer le jour du combat. J'ai abattu tous ses ennemis sur les étendues sableuses de Nédit (shr.n.j hftyw.f $n b$ hr ts $w$ n $N d y t)^{31} »$.

Tout récemment, Mark Smith a relevé le caractère très partiel de l'analyse de Griffiths, qui ignore les sources textuelles évoquées précédemment qui relient Nédit à Bousiris ${ }^{32}$. Si cette mise au point s'imposait, l'argumentation développée par l'auteur doit être examinée de manière critique. En effet, M. Smith sépare d'un côté la «localisation réelle » de Nédit près de Bousiris, et de l'autre les mentions de ce toponyme au cours de la célébration des mystères d'Osiris à Abydos, dans un contexte rituel ${ }^{33}$. Il y aurait donc à distinguer, selon lui, un lieu situé de manière « originelle » dans un mythe fondateur et ses utilisations dans des textes rituels évoquant ce mythe. Cette analyse rencontre des difficultés si l'on prend en compte deux types de sources qui ne peuvent être conçues dans cette optique : d'une part, les textes où un toponyme abydénien se substitue à Nédit dans son emploi habituel; d'autre part, ceux où Nédit est, sans aucune ambiguïté, intégré à la géographie abydénienne. Ce sont deux cas de figure que nous rencontrerons au fil des textes que nous aurons à considérer dans les développements qui suivent.

Dans la notice qu'il a consacrée à Nédit dans le Lexikon der Ägyptologie, Reinhard Grieshammer ${ }^{34}$ a attiré l'attention sur un

30. Citons également un lien établi entre «Celui qui est dans Nedyt» et Bouto en Coffin Texts IV, 383g (Spell 349), mentionné par J. Assmann, Martin Bommas (collab.), Altägyptische Totenliturgien, I, p. 386.

31. Stèle d'Ikhernofret, Berlin 1204, 1. 21.

32. Mark Smith, Following Osiris. Perspectives on the Osirian Afterlife from Four Millenia, Oxford, Oxford University Press, 2017, p. 227-228.

33. Ibid., p. 227, n. $405:$ : Since the mysteries performed there re-enacted the events that followed the murder of Osiris, some descriptions of them do mention Nedit as a place where a part of the action unfolds (...). Thus, in the context of the ritual, Nedit could be transposed to an Abydene setting, but this does not alter the fact that its real location was near Busiris. ».

34. Reinhard Grieshammer, Lexikon der Ägyptologie, éd. Wolfgang Heck, Wolfhart Westendorf, Wiesbaden, Harrassowitz, 1982, t. IV, s.v. « Nedit », col. 372. 
passage du Spell 398 des Textes des Sarcophages ${ }^{35}$, évoquant le combat d'Horus et de Seth à Nédit (var. le district de Nédit), où ce nom est remplacé dans l'une des versions par Abydos (M21C) ${ }^{36}$. L'auteur suggère une solution de «transfert» du toponyme, situé initialement à Bousiris, puis délocalisé à Abydos. Cette seule source ne permet pas néanmoins de conclusions chronologiques si tranchées.

À une date beaucoup plus tardive, le texte des Urkunden VI, énumérant les sacrilèges de Seth, mentionne le «grand crime» perpétré par celui-ci « sur la rive de Thinis » :

«Il a à nouveau perpétré le grand crime, sur la rive de Thinis ( $h r$ $w \underline{d} b n \underline{T} n y)^{37} »$.

L'expression est un décalque de l'évocation, bien connue depuis les Textes des Pyramides, du meurtre d'Osiris « sur la rive de Nédit ${ }^{38}$; le parallèle est encore plus précis avec le passage du Papyrus Jumilhac qui mentionne «le crime qu'avait commis Seth sur la rive de Nédit $(h r w d b N \underline{d} y(t)) »^{39}$. Un lien existe donc naturellement entre les deux lieux ${ }^{40}$, sans qu'il soit aisé de le préciser : synonymie ou symétrie ? Le fait que Seth ici « récidive» $(w h \mathrm{hm})$ dans son crime peut donner une clé d'interprétation ${ }^{41}:$ le meurtre de Thinis reproduirait le forfait initial dans la région bousirite. Une autre interprétation a été proposée par Pascal Vernus qui considère que Thinis, lieu saint, a été substitué à l'évocation d'un lieu néfaste, Nédit, par euphémisme ${ }^{42}$.

35. Coffin Texts V, 128a.

36. Le texte mentionne également un autre lieu, «l'élévation de la terre » (q3jt t3; e.g M3C), mis en parallèle avec Nédit ou Abydos. Sur ce texte, voir Harco Willems, The Coffin of Heqata (Cairo JdE 36418), Louvain, Peeters ( Orientalia lovaniensa analecta », t. 70), 1996, p. 427 et 430-431, n. m et q.

37. Siegfried Schott, Urkunden mythologischen Inhalts, Leipzig, J. C. Hinrichs'sche Buchhandlung («Urkunden des ägyptischen Altertums», t. VI, fasc. 1), 1929, 19, 22-23 (= Urk. VI, 19, 22-23).

38. G. Meurer, Die Feinde des Königs in den Pyramidentexten, p. 122-123.

39. Papyrus Jumilhac XI, 19 ; voir infra au niveau de la n. 48.

40. Voir la discussion de Victoria Altmann, Die Kultfrevel des Seth. Die Gefährdung der göttlichen Ordnung in zwei Vernichtungsritualen der ägyptischen Spätzeit, Wiesbaden, Harrassowitz («Studien zur Spätägyptischen Religion», t. 1), 2010, § 3.1.7, p. 22.

41. Une telle récidive est évoquée dans la formule des stèles d'Horus sur les crocodiles, Texte B, cité supra n. 22.

42. P. Vernus, «Le Mythe d'un mythe : la prétendue noyade d'Osiris », p. 29, n. (18). Voir aussi Pascal Vernus, Dictionnaire amoureux de l'Égypte ancienne, Paris, Plon, 2009, s.v. « Euphémisme », p. 340-341. 


\section{Sur L'UbiQuité de NÉdit}

L'inventaire des mentions de Nédit, même contextualisées, permettrait au mieux de dégager une conviction sur la localisation «canonique» du toponyme, mais n'invite pas à l'imposer de manière péremptoire, a fortiori en prenant en compte la chronologie et la nature disparate des sources. Une autre approche, synchronique celle-là, peut être adoptée en observant comment le toponyme Nédit est utilisé dans l'économie interne de textes considérés comme «mythologiques» selon une définition empirique du terme, à savoir des récits qui évoquent les épisodes de l'existence des dieux. Deux textes sont suffisamment riches d'informations pour nous permettre d'appréhender les contours de cette géographie de Nédit au sein du mythe, à savoir le papyrus Jumilhac et le papyrus Salt 825 .

Le papyrus Jumilhac ${ }^{43}$ est un «manuel mythologique » centré sur les $17^{\mathrm{e}} / 18^{\mathrm{e}}$ nome de Haute-Égypte, mais dont les récits étiologiques locaux s'appuient sur une mythologie d'ampleur nationale, évoquant les lieux et épisodes majeurs de la geste osirienne $^{44}$. Nédit est évoqué comme étant un lieu situé près d'Andjty, dans le Delta, où Anubis retrouve la tête d'Osiris :

43. Outre l'édition de Jacques Vandier, Le papyrus Jumilhac, Paris, CNRS, 1961, on citera parmi les études récentes : Joachim Fr. Quack, "Corpus oder Membra disjecta? Zur Sprach- und Redaktionskritik des Papyrus Jumilhac », Diener des Horus. Festschrift für Dieter Kurth zum 65. Geburtstag, éd. Wolfgang Waitkus, Gladbeck, Pe-We Verlag (« Aegyptiaca Hamburgensia », t. 1), 2008, p. 203-228 ; Sandra Lippert, "L'étiologie de la fabrication des statuettes osiriennes au mois de Khoiak et le Rituel de l'ouverture de la bouche d'après le papyrus Jumilhac », Égypte Nilotique et Méditerranéenne, t. 5, 2012, p. 215-255; Jens Bl. Jørgensen, Egyptian Mythological Manuals. Mythological structures and interpretative techniques in the Tebtunis Mythological manual, the Manual of the Delta and related texts, Université de Copenhague, 2014, p. 242-251, thèse de doctorat en ligne sur le portail academia.edu : <https://independent.academia.edu/ JensJoergensen/Thesis>.

44. Cf. J. B1. Jørgensen, Egyptian Mythological Manuals, p. 243 : «The myth of the head of Osiris also demonstrates that the focus on local myths, rituals and deities does not mean that the manual is wholly unconcerned with national mythology or mythological geography. The myth proceeds from well-known places with a firm tradition in national Osiris mythology, i.e. Nedjyt and Busiris, to the local integration of this myth within local topography. The nome of Dunawy then becomes the focal place for the gathering of the limbs of Osiris who where killed in lower Egypt and whose limbs where dispersed over all of Egypt to be finally recollected in Dunawy. » Sur l'articulation entre mythologies «locale » 
« Anubis alla pour chercher son père Osiris sur la butte des papyrus, sur ce banc de sable de Nédit, à côté d'Andjty, après que le grand naufrage (?) eut lieu dans ce pays. Il trouva la tête auguste de son père sur la colline, trouvé manquant : tout son corps ${ }^{45} »$.

Le même traité nous dit en effet que Seth a décapité Osiris à Nédit :

«Quant au "Lac de la vie": C'est lui - d'après une autre version, parce qu'il est à l'ouest de ce lieu - qu'on appelle "Lac de l'ouest". Anubis et Thot exécutèrent des rituels (?) purs sur la statuette afin de faire parler la tête de son (sic !) père Osiris, pour révéler le(s) lieu (x) où se trouvaient ses membres, à cause du crime qu'avait commis Seth sur la rive de Nédit. Car il commit un crime en face de Nédit quand la tête de ce dieu fut coupée. Anubis se transforma en faucon et $<$ vola $>$ avec elle encore une fois à Hardaï. On le pratique jusqu'à présent ${ }^{46} \gg$.

Alors que ces récits décrivent la décapitation d'Osiris et la découverte de sa tête à Nédit, dans le nome bousirite, en revanche, les récapitulatifs calendériques relatifs aux circonstances de découverte des reliques osiriennes donnent pour lieu de trouvaille de la tête d'Osiris Abydos et non Nédit ${ }^{47}$ : le 19 khoiak, Thot découvre la tête d'Osiris et, sous elle, un scarabée, et le chef divin est alors conservé dans la nécropole d'Abydos ${ }^{48}$. L'apparente contradiction entre ces versions du même mythème suggère là encore que les deux sites renvoient à des épisodes mythologiques proches, mais non identiques, qui précèdent la reconstitution du corps, l'un concernant la décapitation d'Osiris, l'autre l'accès de la tête au statut de relique.

Le papyrus Salt 825 (pBM $10090+$ pBM 10051) ${ }^{49}$, provenant d'Abydos et datable de la $\mathrm{XXVI}^{\mathrm{e}}$ dynastie $^{50}$, contient quant à lui un rituel intitulé « Fin de l'ouvrage (i.e. la fabrication de figurines

et «nationale », voir J. Fr. Quack, « Lokalressourcen oder Zentraltheologie? », p. 5-29.

45. Papyrus Jumilhac X, 22-XI, 2 ; Trad. d'après S. Lippert, «L'étiologie de la fabrication des statuettes osiriennes au mois de Khoiak», p. 217-218.

46. Papyrus Jumilhac XI, 19-22 ; Trad. d'après S. Lippert, op. cit., p. 233.

47. Cf. ibid., p. 250.

48. Papyrus Jumilhac, légendes IV, 1-5 (éd. Vandier, p. 136); légendes V (éd. Vandier, p. 137).

49. Philippe Derchain, Le Papyrus Salt 825, rituel pour la conservation de la vie en Égypte (B.M.10051), Bruxelles, Palais des Académies, 1965, complété par François-René Herbin, «Les premières pages du papyrus Salt 825 », Bulletin de l'Institut français d'archéologie orientale, t. 88, 1988, p. 95-112.

50. Cf. Ursula Verhoeven, Untersuchungen zur späthieratischen Buchschrift, Louvain, Peeters (« Orientalia lovaniensa analecta », t. 99), 2001, p. 287. 
osiriennes) » (phwy kzt), conservé partiellement ; le début du texte est occupé par un récit à caractère mythologique racontant le temps de la création du monde, la naissance d'Osiris, son enfance auprès de ses parents Chou et Tefnout, son installation à Ta-our jusqu'à son meurtre par Seth. Il localise le meurtre d'Osiris par Seth dans le Hat-djefaou, sous l'arbre arou $^{51}$, à Nédit :

«[... (c'est un chemin) qu'ils firent en direction de Nédit, qui s'appelle (aussi) Hat-djefaou, [... l'ass]emblée de Rê, les Ba d'Héliopolis, Ioun le Bien-Aimé... [...] Nédit ${ }^{52} »$.

(après l'évocation de la naissance et la jeunesse d'Osiris) :

«Il arriva à un sol. Alors Osiris dit: "combien grande (our) est cette terre $(t a)$ !" On l'appelle Ta-our à cause de cela jusqu'à ce jour. Osiris s'y trouvait très bien; il fut entendu par Seth, et Seth vint en hâte ; il arriva contre "l'ennemi" d'Osiris à l'intérieur de Nédit dans Hat-djefaou, sous l'un des arbres dont le nom est ârou, le $1^{\text {er }}$ mois de la saison-akhet, le $17^{\mathrm{e}}$ jour. Il commit un grand crime contre son ennemi, et fit qu'il dérivât sur l'eau. Alors Noun entra dans une extrême colère à cause de cela ; il monta pour cacher ses mystères et le remodela en lui. Il fut entendu par Rê ; il vint en hâte [...] Chou et Tefnout pleurèrent abondamment; ils dirent : "Malheur !... (?) Mensonge !... (?)" 53 ».

Le Hat-djefaou, devenu ultérieurement Hout-djefaou (Châteaudes-aliments $)^{54}$, est un lieu sacré osirien dont l'archétype se trouve

51. Sur l'arbre ârou, voir Pierre Koemoth, Osiris et les arbres. Contribution à l'étude des arbres sacrés de l'Égypte ancienne, Liège, Centre informatique de Philosophie et Lettres (« Aegyptiaca Leodiensia », t. 3), 1994, p. 183-193 ; Isabelle Régen, «À propos d'une mention de l'arbre ' $r w$ dans le chapitre 151A du Livre des Morts ", Encyclopédie religieuse de l'univers végétal. Croyances phytoreligieuses de l'Égypte ancienne, éd. Sydney H. Aufrère, Montpellier, Université Paul Valéry («Orientalia Monspeliensia », t. 11), 2001, t. 2, p. 299-318; Jean-Claude Goyon, Le recueil de prophylaxie contre les agressions des animaux venimeux du Musée de Brooklyn, Wiesbaden, Harrassowitz ( Studien zur Spätägyptischen Religion », t. 5), 2012, p. 57-58 ; Susanne Töpfer, Das Balsamierungsritual. Eine (Neu-)Edition der Textkomposition Balsamierungsritual (pBoulaq 3, pLouvre 5158, pDurham 1983.11 $+p$ St. Petersburg 18128), Wiesbaden, Harrassowitz (« Studien zur Spätägyptischen Religion », t. 13), 2015, p. 164, n. (i). L'arbre joue le rôle de protecteur de la sépulture d'Osiris mais participe également à sa renaissance (I. Régen, op. cit., p. 309-311).

52. Papyrus BM 10090, page X + II, 1-3 ; Fr.-R. Herbin, «Les premières pages du papyrus Salt $825 »$, p. 102 et pl. VII.

53. Papyrus BM 10090, page $X+V, 1-6$; Fr.-R. Herbin, « Les premières pages du papyrus Salt 825 », p. 103 et pl. VI. Pour la traduction, nous avons tenu compte de P. Vernus, «Le Mythe d'un mythe : la prétendue noyade d'Osiris », p. 21-22 (21).

54. Voir dernièrement S. Töpfer, Das Balsamierungsritual, p. 132-133, n. (ax). Pour un réexamen de toutes les attestations, voir Laurent Coulon, $\mathrm{La}$ chapelle d'Osiris Ounnefer Neb djefaou, un reposoir du "fétiche" abydénien à Karnak. Publication épigraphique et analyse du programme décoratif, avec des 
dans la mythologie abydénienne. Le nom de Château-des-aliments est en effet donné à la butte sacrée d'Abydos dans le manuel géographique de Tebtynis ( $\mathrm{II}^{\mathrm{e}} \mathrm{s}$. apr. J.-C.) $)^{55}$. Dans les liturgies funéraires tardives, ce toponyme est également fréquemment utilisé en corrélation avec des désignations d'Abydos ou de ses désignations alternatives ou métonymiques (Ou-Peker dans le Rituel de l'Embaumement ${ }^{56}$, la-place-du-scarabée dans la liturgie funéraire du papyrus Harkness ${ }^{57}$ ). Il en existe néanmoins également dans d'autres localités d'Égypte : Coptos $^{58}$, le district de Dounâouy dans le $17^{\mathrm{e}} / 18^{\mathrm{e}}$ nome de Haute Égypte ${ }^{59}$, à Xoïs ${ }^{60}$, et possiblement à Karnak, où la chapelle d'Osiris Ounnefer Neb Djefaou répondrait à la définition d'un «Château-des-aliments », désignation qui ne se trouve pas néanmoins employée pour la désigner.

De manière assez singulière, les deux extraits du papyrus Salt 825 cités précédemment situent Nédit dans le Hat-djefaou, à Abydos. Le deuxième extrait lève tout doute sur le fait qu'il s'agit bien là du lieu où est perpétré le crime envers Osiris. La « rive maudite », point de départ de la dérive du corps osirien, se trouve indubitablement attachée au nome thinite et la géographie mythologique mobilisée ici fait d'Abydos le théâtre unique du mythe osirien. Deux hypothèses sont envisageables: soit le toponyme Nédit était déjà perçu comme un toponyme abydénien,

contributions de Cyril Giorgi et Aleksandra Hallmann, dessins d'Anna Guillou, à paraître à l'Institut français d'archéologie orientale.

55. Papyrus Carlsberg 182.2, L 21 ; Jürgen Osing, Hieratische Papyri aus Tebtunis, Copenhague, Museum Tusculanum Press ( Carsten Niebuhr Institute Publications », t. 17 ; «The Carlsberg Papyri », t. 2), 1998, t. I, p. 250 et pl. 25.

56. S. Töpfer, Das Balsamierungsritual, p. 117.

57. Col. V, 9. Cf. Mark Smith, Papyrus Harkness (MMA 31.9.7), Oxford, Griffith Institute, 2005, p. 79 ; id., Traversing Eternity. Texts for the Afterlife from Ptolemaic and Roman Egypt, p. 296.

58. Philippe Derchain, Les impondérables de l'hellénisation. Littérature d'hiérogrammates, Bruxelles-Turnhout, Fondation égyptologique Reine ÉlisabethBrepols (« Monographies Reine Élisabeth», t. 7), 2000, p. 49-53, pl. VI.

59. Papyrus Jumilhac IX, 20-22 (voir infra n. 65). Le "Château-desaliments » a été préalablement mentionné dans la liste des toponymes du district de Dounaouy (VII, 22).

60. Opet, $\mathrm{n}^{\circ} 191$; traductions: Ivan Guermeur, Les cultes d'Amon hors de Thèbes. Recherches de géographie religieuse, Turnhout, Brepols (« Bibliothèque de l'École des Hautes Études-Sciences Religieuses», t. 123), 2005, p. 171 ; Aurélie Paulet, Thesaurus Linguae Aegyptiae, Ptolemaic and Roman Hieroglyphic Texts, Karnak, Opettempel. Transcription et traduction (http://aaew.bbaw.de/tla/ ; dernière consultation le 15/12/2013). 
soit le récit mythologique a voulu relocaliser l'ensemble du parcours d'Osiris dans un paysage local, pour créer une unité de $\operatorname{lieu}^{61}$. Faut-il lier ce resserrement topographique à la conception qui se développe à l'époque tardive selon laquelle les humeurs $(r \underline{d} w)$ que la putréfaction du cadavre dégage sont des reliques à part entière ${ }^{62}$ et que ce sont ces humeurs d'Osiris qui fertilisent la terre du Château-des-aliments pour permettre ensuite de générer la prospérité pour le pays, comme l'exposera très clairement une notice du papyrus Jumilhac ${ }^{63}$ ? Insérer Nédit dans le Hat-djefaou permet de circonscrire le meurtre dans un site qui, érigé en tombeau ritualisé ${ }^{64}$, peut engager immédiatement le cadavre et ses émanations dans un cycle de revitalisation.

Cependant, même si Nédit semble ainsi absorbé dans l'univers abydénien, il est à noter que, dans une autre séquence du papyrus Salt 825, la dualité Bousiris / Abydos est réactivée. Il s'agit d'un passage à vocation étiologique où sont décrits les lieux d'installation des principaux dieux, là où ils ont laissé tomber leur sueur :

«La terre du Château-du-Benben (= Héliopolis); la terre d'Iyt (= Létopolis). Râ s'y est installé, sa sueur y est tombée. La terre de Tjenenet (= Memphis), Ptah s'y est installé ; sa sueur y est tombée. La terre de Bousiris, la terre du Hat-djefaou, Osiris s'y est installé ; sa sueur y est tombée ${ }^{65} \gg$.

61. $\mathrm{Ph}$. Derchain invoque les «nécessités du rituel» pour justifier la transposition de Nédit au voisinage d'Abydos (Le Papyrus Salt 825, p. 45), mais il s'agit d'une explication qui ne rend pas compte, nous semble-t-il, de la logique de construction du discours mythologique ici.

62. Cf. Laure Pantalacci, «Une conception originale de la survie osirienne d'après les textes de Basse Époque », Göttinger Miszellen, t. 52, 1981, p. 57-64.

63. Papyrus Jumilhac IX, 20-22: «Quant au Château-des-aliments $(h(w)$ $t$ - $d f_{3} w$ ), (c'est) le temple où sont produites les nourritures à partir des humeurs sorties d'Osiris - pour répéter : ce que l'on dit du derrière de ce dieu - comme quelque chose qu'y ont apporté les enfants d'Horus ». Trad. d'après S. Lippert, «L'étiologie de la fabrication des statuettes osiriennes », p. 225-226; néanmoins, notre traduction ne reprend pas son hypothèse de lecture $k f_{3} t$ « écoulement » à la place de $p h w y$ « derrière ».

64. Les plantes-nbh qui, comme le précise le Papyrus Salt 825, proviennent d' « Osiris dans Nedyt » (papyrus BM 10051, XV, 4-5 = Ph. Derchain, Le papyrus Salt 825, p. 143 et pl. 16*), sont les marqueurs de cette sépulture donnée au corps qui est le point d'appui du cycle de renaissance. Sur ces plantes-nbh (et leur origine bousirite), voir P. Koemoth, Osiris et les arbres, p. 100-112.

65. Papyrus BM 10051, III, 5-6. Voir Ph. Derchain, Le Papyrus Salt 825, I, p. 137-138, II, pl. 3*. 
La lecture «La terre de Bousiris » a été établie par Ursula Verhoeven ${ }^{66}$, alors que l'édition de $\mathrm{Ph}$. Derchain portait la leçon «Abydos », celui-ci ayant vraisemblablement été influencé par le caractère fortement abydénien de l'ensemble des récits mythiques. En fait, le dualisme des lieux saints osiriens est finalement réaffirmé, même si la geste osirienne est ici entièrement focalisée sur les structures locales d'Abydos.

En définitive, s'il ne faut certainement pas céder à un relativisme total dans l'appréhension de la géographie mythologique osirienne ${ }^{67}$, il est essentiel de saisir toute la complexité d'y associer une géographie «réelle» et des traditions locales. La dualité originelle entre Abydos et Bousiris a été érigée en dualisme systématique, dans lequel chaque centre théologique s'est constitué un univers «complet» en miroir de l'autre - tout au moins peut-on en être convaincu pour Abydos, bien mieux documenté que Bousiris. Comme les exemples du papyrus Jumilhac et du papyrus Salt 825 le montrent, Nédit peut être tantôt un toponyme deltaïque, symétrique à Abydos, tantôt un élément du paysage religieux abydénien. Il est à déplorer que si peu de sources nous soient accessibles concernant la théologie bousirite pour pouvoir dire si, en retour, un Château-des-aliments y avait été acclimaté... Dans l'état actuel de nos connaissances, il est indéniablement très difficile de «hiérarchiser» les deux centres osiriens et de déterminer précisément comment s'est élaborée la géographie du mythe osirien.

En tout état de cause, la collecte des sources a montré la récurrence très fréquente de la caractérisation du lieu de la mort

66. U. Verhoeven, Untersuchungen zur späthieratischen Buchschrift, p. 288. Voir aussi Frank Feder, Thesaurus Linguae Aegyptiae, Papyrus BM 10090+10051 (Papyrus Salt 825) ( "Rituel pour la conservation de la vie»). Transcription et traduction (http://aaew.bbaw.de/tla/ ; dernière consultation le 25/07/2018).

67. Tel que celui de Vincent A. Tobin, « Divine conflict in the Pyramid Texts », Journal of the American Resarch Center in Egypt, t. 30, 1993, p. 103 : " In any event, the actual place of Osiris' death is not important as a specific geographical location. A consideration of the event as a mythic symbol would imply that the location of both Nedit and Ghsty was wherever the myth was ritually and cultically performed. » 
d'Osiris comme une « rive » $(w \underline{d} b)$ ou un « banc sableux » $(\underline{t} s w)$, entre autres dénominations plus rares. Outre leur lien avec le Nil, qui joue un rôle central dans l'épisode de la dérive du cadavre, ces espaces sont associés aux notions d'instabilité $e^{68}$ et de danger sournois $^{69}$ qui renforçaient naturellement le caractère sinistre du lieu... et l'insaisissabilité du toponyme.

laurent.coulon@ephe.sorbonne.fr

68. Voir l'analyse récente du terme $w \underline{d} b$ comme « bande de terre temporaire et mouvante » par Katia Novoa, «La rive $w \underline{d} b$. Étude lexicographique », Égypte. Afrique \& Orient, t. 88, 2017-2018, p. 35-44.

69. Sur le banc de sable-tisw comme danger fluvial et manifestation d'ApophisSeth s'opposant à la barque de Rê, voir Adolphe Gutbub, «La tortue animal cosmique bénéfique à l'époque ptolémaïque et romaine », Hommages à Serge Sauneron, Le Caire, Institut français d'archéologie orientale («Bibliothèque d'Étude », t. 81), 1979, t. I, p. 420-421; Sydney H. Aufrère, L'univers minéral dans la pensée égyptienne, Le Caire, Institut Français d'Archéologie Orientale («Bibliothèque d'Étude», t. 105), 1991, p.672-673; sur la perception des étendues sableuses dans le paysage égyptien, voir aussi Julie Misuriello, «La double perception du sable en Égypte ancienne», Techniques \& Culture [En ligne], 61 | 2013, mis en ligne le 15 décembre 2016, consulté le 26 juillet 2018. URL : http://journals.openedition.org/tc/7212 ; DOI : 10.4000/tc.7212. 